\title{
Celebration of QED's half-century
}

A badly constructed yet fascinating account of the origins of quantum electrodynamics suggests the need for a way of capturing both the spirit of an heroic age and the later history of the concepts that it spawned.

THIS year is thick with fiftieth anniversaries, mostly because the European phase of the Second World War ended 50 years ago. But 1945 also marked the return to academic laboratories of people who had spent the war at places such as Los Alamos, the Radiation Laboratory at MIT and its outposts (most significantly that at Columbia in New York), not to mention Chalk River and Britain's equivalent of the RadLab at Malvern.

One of those to return from the war was the late Maurice Pryce, one of the first of Oxford's professors to be appointed then. With his formidable reputation in theoretical physics, his knack for dressing well and his marriage to Max Born's daughter Margaret, he was a focus of attention.

Pryce's first course on quantum mechanics, begun in October 1946, was therefore filled with the curious as well as the eager. In the event, the course was an intelligent scamper through Dirac's great book The Principles of Quantum Mechanics. The importance of the inside information that Pryce kept pressing on his audience went largely unrecognized; he was full of exhortations to pay more attention to the energy levels of the hydrogen atom. He mentioned a fellow called Lamb, then at Columbia. Few appreciated that he was pointing to an inside track to fame and fortune.

The hydrogen problem was the springboard on which the great revolution of quantum electrodynamics was mounted. What follows is mostly about a remarkable book on that formative experience for the research community, $Q E D$ and the Men Who Made It, by Silvan S. Schweber, a physicist turned historian who has gone back to physics to celebrate QED (Princeton University Press, 1994, \$99.50 (hbk), $\$ 39.50$ (pbk)).

Predictably, the names cited on the cover are Dyson, Feynman, Schwinger and Tomonaga (in alphabetical order), but Schweber casts his net wider that that. Thus he staunchly defends the value of the contributions made in the 1920 s by Pascual Jordan, one of the authors (with Born and Heisenberg) of the famous and monumental article still called the Dreimännerarbeit (Zeit. Phys. 35, 557-615; 1926).

Jordan seems to have contributed to that landmark paper the final section in which a vibrating string was a one-dimensional analogue of the radiation field; each vibrational mode becomes the equivalent of one of the oscillator modes into which the radiation field can be decomposed. Schweber guesses that Jordan's contribution would now be more honoured had he not become a member of the Nazi party and, separately, taken to sports such as psychology, all the while hampered in communicating with others by a crippling stammer.

If that seems trite, the same is also true of the biographical sketches with which Schweber's book is loaded. Was Schwinger's notorious habit of working only at night a simple consequence of his "shyness", and his fear of being "dominated" by pushy individuals? Schwinger (now, sadly, dead) would probably have a more interesting tale to tell. The anecdotal information that Schweber has painstakingly gathered is in many ways a distraction.

It is, of course, interesting and important to know what people such as Rabi and Oppenheimer did to create the intellectual climate in which the problems in electrodynamics were solved not once, but twice (by Schwinger and Feynman). But Schweber is a laundry-list biographer; if he has a piece of information, he puts it in, often by quoting at length from original documents. (There is also a laundry-list of a kind - the budget for a conference at Shelter Island, off the north coast of Long Island, in the spring of 1947 , estimated to cost $\$ 3,150$.) His discussion of how the habit of solving practical problems for the war changed the temperament of physics in the United States is similarly unconvincing. The result is a book (at 732 pages) too long by half.

But one should not be too harsh. Although Schweber deals with physics in much the same way, reproducing passages from crucial articles and whole letters from the likes of Pauli and Dirac, the result is a kind of source-book, even a scrapbook, for those who want to know more about the revolution that set the trend and the pace for the breathtaking development of the rarefied end of theoretical physics in the past half-century. And Schweber, like Pryce all those years ago, is right to emphasize the importance of what Lamb did.

The issue, half a century ago, was the suspected difference of energy between the two possible configurations of the first excited state of hydrogen. An electron in the ground state must have zero angular momentum (and its wavefunction must be spherically symmetrical), but the first excited state (with principal quantum number 2) allows states $S$ and $P$ in which the angular momentum is either 0 or 1 respectively, and called $2 S^{1 / 2}$ and $2 P^{1 / 2}$. Simple wave mechanics shows the two states to be degenerate. Lamb's experiment, a tour de force for the time, demonstrated an energy difference of about $1,000 \mathrm{MHz}$, with the $S$ state at the higher energy.

Remarkably, at the same laboratory at Columbia, at the same time, Rabi and his associates uncovered another crucial piece of evidence: the magnetic moment of the electron is not exactly what it should be (1 Bohr magneton, the magnetic moment associated with one unit of angular momentum in an electron orbit), but some 0.12 per cent greater. These were the data with which the score of theoreticians gathered for the Shelter Island conference in 1947 were challenged.

Schweber's anecdotal method works to his advantage in reconstructing that event, largely with the help of notes kept by bystander participants. The penny seems to have dropped quickly. In each case, the explanation must be the interaction between the electron and the radiation field. The book tells how Hans Bethe made a non-relativistic calculation of the Lamb shift on the train to Schenectady, bound for a few days consulting for General Electric.

Instead of following the then standard practice of ignoring the infinite quantities notoriously obtained in calculations such as this, Bethe followed the advice of Kramers (from Leyden, who had been at Shelter Island) that the effects of the radiation field on the apparent mass of the electron should be counted as a part of its observed mass. That was the first application of the ungainly techniques of renormalization by which field theories are still beset.

From there, it is a helter-skelter tale. Half a dozen groups were busy with relativistic calculations of the Lamb shift; the unluckiest were Weisskopf and French, at MIT, who got the correct result but delayed publication because others told them of discordant conclusions. (There is a moral there.) Schwinger produced a general theory, Feynman developed his other scheme and Dyson proved that the two views were equivalent.

The three years from 1945 to 1948 set the style for everything that has followed since in the understanding of matter, radiation and the link between them. It is an heroic episode that amply deserves celebration. But Schweber could at least have guided his readers towards the uses afterwards made of quantum field theory in the understanding of other components of matter than electrons. That is also an heroic tale.

John Maddox 